\title{
Relação entre força muscular e capacidade funcional em pacientes com doença arterial obstrutiva periférica: um estudo piloto
}

\section{Relationship between muscular strength and functional capacity in patients with peripheral occlusive arterial disease: a pilot study}

Danielle Aparecida Gomes Pereira', Bruna Marques Araújo Faria², Rayssa Amaral Machado Gonçalves², Vivielle Brígida Ferreira Carvalho², Karoline Oliveira Prata², Poliana Soares Saraiva ${ }^{2}$, Túlio Pinho Navarro³, Inácio Cunha-Filho ${ }^{4}$

\begin{abstract}
Resumo
Contexto: As informações sobre o grau de correlação entre força muscular e capacidade funcional em pacientes com doença arterial obstrutiva periférica (DAOP) são inconsistentes, além de nem sempre apresentarem protocolos que relacionem apropriadamente medidas de força muscular com desempenho.

Objetivo: Estabelecer o nível de correlação entre força muscular e capacidade funcional em pacientes fisicamente ativos com DAOP.

Métodos: Doze pacientes com diagnóstico da doença e presença de claudicação intermitente participaram do estudo. Todos realizaram testes de força muscular e de caminhada. Grau de correlação entre força muscular e capacidade funcional foi avaliado pela correlação de Pearson.

Resultados: Dos 12 voluntários incluídos, 10 eram do sexo masculino e 2 do sexo feminino. Os participantes tinham média de idade de $63 \pm 11$ anos. Houve alta correlação $(r=0,872 ; p=0,0001)$ entre distância percorrida no teste de deslocamento bidirecional progressivo (TDBP) e carga alcançada no teste de resistência máxima. Não se observou correlação entre distância percorrida no TDBP e tempo gasto para realização das cinco flexões plantares no teste de ponta de pé.

Conclusão: O desempenho funcional em um grupo de pacientes com DAOP, em sua maioria fisicamente ativos, foi fortemente correlacionado com força muscular de extensores de joelho, mas não com desempenho observado funcionalmente pelo teste de ponta de pé. Futuros estudos são necessários para avaliar se força muscular de flexores plantares, mensurada de forma específica e isolada, correlaciona-se com função em pacientes com a doença.
\end{abstract}

Palavras-chave: Claudicação intermitente; força muscular; isquemia.

\begin{abstract}
Background: Information on the degree of correlation between muscular strength and functional capacity in subjects with peripheral obstructive arterial disease (POAD) is inconsistent and the available studies use protocols that do not adequately correlate measurements of muscular strength with performance.

Objective: to correlate muscular strength and functional capacity in physically active subjects with POAD.

Methods: Twelve subjects with PAOD and intermittent claudication were enrolled in the study. All subjects performed muscular strength and walking tests. Pearson's correlation was used to determine the level of association between these variables.

Results: Twelve subjects, 10 males and 2 females, were included in the study. The mean age was $63 \pm 11$ years. There was a strong correlation $(r=0.872$; $p=0.0001$ ) between the distance walked in the shuttle walk test (SWT) and the maximum load at the maximum strength test. However, there was no correlation between the distance reached in the SWT and the time spent to carry out five plantar flexions in the tiptoe test.

Conclusion: The functional performance of a group of individuals with PAOD - most of them physically active - was strongly correlated with knee extension muscular strength, but not with performance observed at the tiptoe test. Further studies must be carried out to assess whether the muscle strength of plantar flexion, measured specifically and separately, is correlated with function in PAOD patients.
\end{abstract}

Keywords: Intermittent claudication; muscle strength; ischemia.

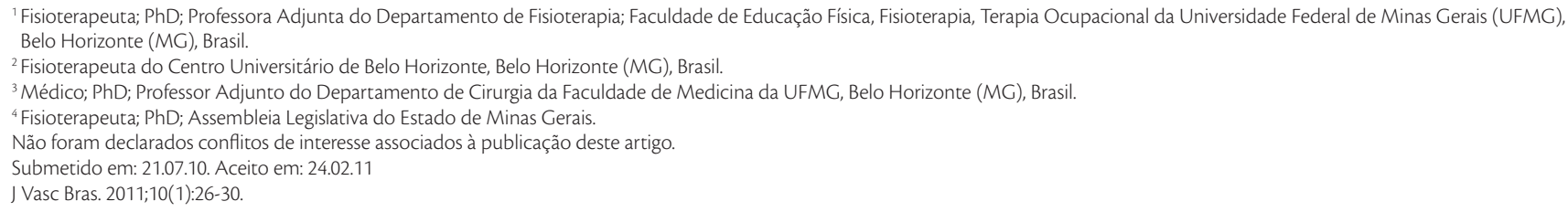




\section{Introdução}

Nas últimas três décadas, a doença arterial obstrutiva periférica (DAOP) tem sido uma das principais causas de morbidade entre a população idosa ${ }^{1-8}$. Seu principal sintoma é a claudicação intermitente $(\mathrm{CI})^{7-10}$, a qual é descrita como presença de dor nos membros inferiores, queimação ou câimbra, ocorrendo durante a atividade física e aliviando com o repouso ${ }^{11}$. A CI compromete as atividades físicas diárias, limita a capacidade funcional e afeta negativamente a qualidade de vida dos pacientes com a doença $a^{2,7}$.

A DAOP está também associada a mudanças crônicas na morfologia e na função da musculatura afetada ${ }^{12}$. As principais alterações da musculatura esquelética consistem em denervação muscular, diminuição da velocidade da condução nervosa, atrofia seletiva de fibras musculares e alterações na atividade enzimática ${ }^{2,6,8,10,12,13}$. Alguns estudos relatam que pacientes com doença isquêmica desenvolvem atrofia e diminuição seletiva de fibras tipo II em relação ao tipo I, o que pode contribuir para disfunção muscular ${ }^{10,12}$. Outros autores relatam maior perda de fibras tipo $\mathrm{I}^{1}$. Existem evidências de que, em conjunto, tais alterações associam-se a uma menor força muscular e pior funcionalidade nos pacientes acometidos pela doençą, ${ }^{2,6,10,14}$. Além disso, sugere-se que o declínio observado na força muscular pode também ser influenciado pelo sedentarismo frequentemente desses pacientes ${ }^{7,12,13}$.

Informações acerca do grau de associação entre força muscular e funcionalidade nos pacientes com DAOP são escassas. Alguns autores referiram que a redução da força muscular em membros inferiores (MMII) é fator preponderante nas limitações funcionais em pacientes com $\mathrm{DAOP}^{2,6,10,14}$, porém não há relatos precisos de quantificação dessa relação. Outros encontraram correlações fracas entre força e função e relataram que os demais aspectos do paciente merecem ser investigados para explicar a capacidade de deambular ${ }^{10}$.

Assim, as informações sobre o grau de correlação entre força muscular e capacidade funcional na DAOP são inconsistentes, além de nem sempre apresentarem protocolos que relacionem apropriadamente as medidas de força muscular com desempenho. Além disso, resultados sobre o treinamento muscular em pacientes com DAOP são escassos em comparação com o exercício aeróbico, permanecendo ainda contraditórios ${ }^{15-17}$, e a indicação desse tipo de exercício não é padrão na reabilitação vascular. A avaliação da relação entre força e função pode auxiliar na tomada de decisão clínica, incluindo ou não o treinamento de força muscular na reabilitação. O objetivo deste trabalho foi, portanto, estabelecer o nível de correlação entre força muscular e capacidade funcional em pacientes com DAOP.

\section{Métodos}

Trata-se de um estudo transversal, aprovado pelo Comitê de Ética em Pesquisa do Centro Universitário de Belo Horizonte, sob o parecer n. 031/2006.

\section{Participantes}

Foram selecionados para o estudo 12 pacientes com DAOP - independentemente de sexo, idade, etnia e estilo de vida - com presença de CI. Incluíram-se pacientes com índice tornozelo-braço (ITB) menor que 0,9 , pertencentes à classe II de Fontaine (presença de CI). Os critérios de exclusão foram: ITB $>0,9$; presença de dor em repouso; presença de doenças ou complicações que impedissem a realização de exercício, tais como insuficiência cardíaca descompensada, angina instável, arritmias ou sinais de instabilidade hemodinâmica; pacientes com acidente vascular encefálico que exibissem comprometimento neurológico na marcha. A amostra foi recrutada por conveniência no setor de doenças vasculares periféricas do Centro Universitário de Belo Horizonte, onde se realizou o estudo, e em consultórios médicos, a partir de indicações de angiologistas. Nenhum dos voluntários contatados apresentava dor isquêmica em repouso ou doenças ortopédicas, neurológicas ou cardíacas que fossem contraindicadas para a realização dos testes. Todos os voluntários assinaram termo de consentimento livre e esclarecido.

\section{Medidas}

Examinadores devidamente treinados realizaram os testes após um estudo prévio para avaliação da confiabilidade das medidas. A reprodutibilidade tanto intraexaminador quanto interexaminador foi alta $(r>0,8 ; \mathrm{p}<0,05)$. Coletaram-se os dados do teste de caminhada (teste de deslocamento bidirecional progressivo - TDBP) e dos testes de força muscular com intervalo mínimo de 24 horas e máximo de 1 semana, em ordem de aplicação aleatória.

Selecionou-se o TDBP, ou Shuttle Walk Test, com o intuito de mensurar a capacidade funcional ${ }^{18}$. Nesse teste, realiza-se um percurso com distância de $9 \mathrm{~m}$, demarcado por dois cones, perfazendo uma distância total de $10 \mathrm{~m}$. O teste é composto por 12 estágios e cada um tem a duração de 1 minuto. O número de percursos aumenta a cada estágio, sendo o primeiro composto por 3 percursos e o último composto por 14. A velocidade é determinada por sinais de áudio, sendo aumentada a cada estágio ${ }^{18}$. O teste é interrompido quando o paciente não consegue alcançar o ponto 
de retorno estabelecido por duas vezes consecutivas. Dois examinadores aplicaram o TDBP e as variáveis de interesse registradas foram distância percorrida e velocidade máxima alcançada no teste.

Dois examinadores treinados realizaram dois testes de força muscular. Entre os dois testes, determinou-se um período de repouso de cinco minutos. Escolheu-se o teste de ponta do pé por avaliar a musculatura comumente afetada em pacientes com DAOP. Realizou-se o teste com o paciente de pé descalço com a mão direita apoiada à frente na parede e com semiflexão de cotovelo para auxílio na manutenção do equilíbrio ${ }^{19}$ (Figura 1). Previamente à realização do teste, o participante realizou uma flexão plantar até o ponto de apoio das articulações metatarso-falangeanas e essa altura foi demarcada pelo examinador por meio da haste de um equipamento fixado na parede ${ }^{19}$. Informou-se ao participante que, durante a realização do teste, deveria sempre alcançar aquela altura previamente estipulada ${ }^{19}$. O teste propriamente dito consistiu em cronometrar, em segundos, o tempo gasto para realizar cinco flexões plantares completas o mais rápido possível. Realizou-se também o teste de uma resistência máxima (1RM) no banco extensor para avaliação de força dos extensores de joelho (Figura 1). A coluna vertebral do participante deveria estar apoiada e o encosto regulado para manter a distância de $5 \mathrm{~cm}$ entre a fossa poplítea e a extremidade do banco. O participante realizou extensões completas do joelho direito (padronizado para todos) com carga inicial de $5 \mathrm{~kg}$ e com incremento de $1 \mathrm{~kg}$ até que conseguisse realizar uma única repetição. A carga alcançada nessa repetição foi definida como 1RM. O examinador demonstrou ao participante uma repetição para a realização correta do teste.

\section{Análise estatística}

Apresentou-se a estatística descritiva como média \pm desvio padrão. Realizou-se o teste de Shapiro-Wilk para avaliar a distribuição dos dados. Avaliou-se a correlação entre força muscular e capacidade funcional pela correlação de Pearson. Requereu-se um valor de alfa menor ou igual a 5\% para significância estatística.

\section{Resultados}

Dos 12 voluntários incluídos, 10 eram do sexo masculino, 58,3\% eram hipertensos, 16,7\% apresentavam diabetes, $41,7 \%$ eram tabagistas e $75 \%$ realizavam atividade física regular. Os participantes tinham média de idade de $63 \pm 11$ anos, índice de massa corporal (IMC)

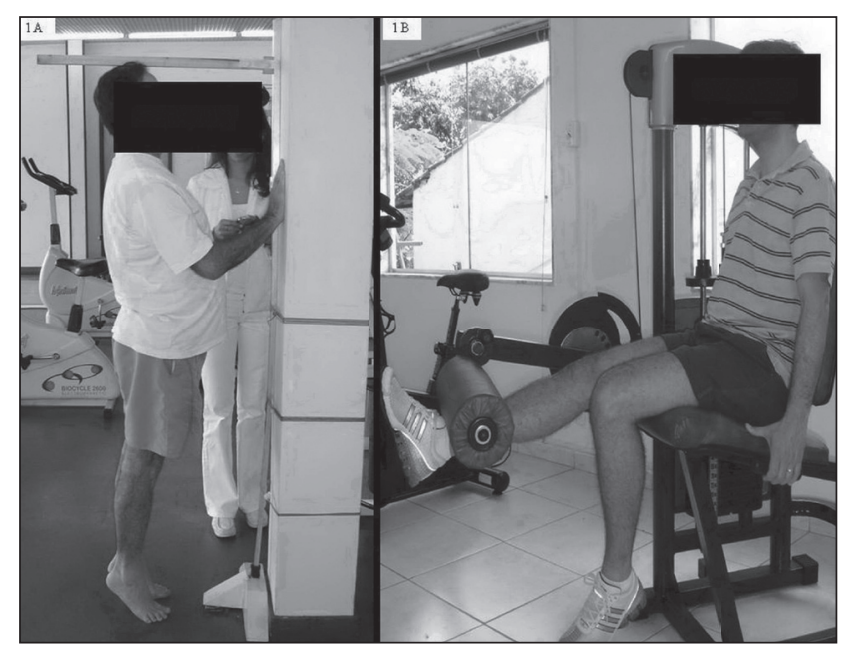

Figura 1 - Testes de força muscular. A: teste de ponta do pé; B: teste de $1 \mathrm{RM}$ no banco extensor.

médio de 25,53 \pm 3,31 (sobrepeso segundo a Organização Mundial de Saúde - OMS) e ITB médio de 0,73 \pm 0,14. Somente dois voluntários não faziam uso de medicação. Antiplaquetário, diurético, hipoglicemiante e vasodilatador estavam entre as classes de drogas mais utilizadas pelos participantes do estudo.

Todos os participantes foram capazes de realizar os testes de TDBP, de 1RM e de ponta do pé sem intercorrências. A média de distância percorrida no TDBP foi de 410,83 \pm 168,49 m, alcançando-se uma velocidade máxima de 93,33 $\pm 17,75 \mathrm{~m} /$ minuto. O tempo médio gasto para a realização do teste de ponta do pé foi de $8,19 \pm 3,84$ segundos, e a carga média alcançada no teste de $1 \mathrm{RM}$ realizado com os extensores de joelho foi de $13,75 \pm 6,3 \mathrm{~kg}$.

Houve alta correlação entre distância e velocidade percorrida no TDBP com a carga alcançada no teste de $1 \mathrm{RM}, \mathrm{r}=0,872 ; \mathrm{p}=0,0001$ e $\mathrm{r}=0,838 ; \mathrm{p}=0,001$, respectivamente. Não se observou correlação significativa entre distância percorrida no TDBP e tempo gasto para a realização das cinco flexões plantares no teste ponta do pé (Tabela 1).

Tabela 1 - Correlação entre força muscular e capacidade funcional

\begin{tabular}{lcc}
\hline & 1RM $^{*}$ & T_PP \\
\hline D_TDBP & $r=0,872^{* *}$ & $r=-0,217$ \\
V_MAX & $r=0,838^{* *}$ & $r=-0,188$ \\
n & 12 & 12 \\
\hline
\end{tabular}

r: coeficiente de correlação de Pearson; D_TDBP: Distância alcançada no teste de deslocamento bidirecional progressivo; $V \_$MAX: velocidade máxima alcançada no teste de deslocamento bidirecional progressivo; *carga alcançada no teste de 1 RM realizado com os extensores de joelho; T_PP: Tempo, em segundos, gasto para a realização do teste de ponta de pé; ${ }^{* *} p<0,05$ 


\section{Discussão}

No presente estudo, em pacientes com DAOP, encontrou-se alta correlação $(r=0,872)$ entre força muscular avaliada pelo teste de $1 \mathrm{RM}$ realizado com extensores de joelho e capacidade funcional mensurada pela distância percorrida no TDBP. O TDBP exige incremento progressivo de velocidade, e a força muscular de extensores de joelho pode ser um fator que contribui para o desempenho durante a sua realização ${ }^{20}$. Essa hipótese é reforçada observando-se a correlação encontrada entre 1RM e velocidade máxima alcançada no TDBP $(r=0,838)$.

A variância compartilhada, considerando-se o TDBP e a carga alcançada no teste de 1RM neste estudo, foi de $0,76\left(\mathrm{r}^{2}\right)$, podendo-se inferir, então, que $76 \%$ da variação no TDBP podem ser explicados pelo desempenho no teste de 1RM. Como a indicação de exercício de força muscular não é padrão na reabilitação vascular, conhecer a relação entre força e função pode auxiliar na tomada de decisão clínica, incluindo ou não outras formas de treinamento na abordagem de pacientes com DAOP.

Comparando os dados obtidos neste estudo com a literatura, encontramos resultados semelhantes, mas alguns com grau de correlação menor entre força muscular e capacidade funcional. Mcdermott et al..$^{21}$ verificaram em seu estudo que a potência muscular de extensores de joelho relacionou-se diretamente com a capacidade funcional avaliada por um questionário de desempenho e testes de caminhada; porém, não se demonstrou a correlação entre essas variáveis.

Atkins e Gardner ${ }^{2}$, avaliando a relação entre força muscular funcional e gravidade na DAOP, demonstraram fraca correlação $(r=0,297)$ entre o desempenho no teste de senta e levanta e distância percorrida em teste de caminhada de 6 minutos. Os autores do estudo não caracterizaram os pacientes quanto ao nível de atividade física, não se especificando a proporção de sedentários e treinados na população avaliada. Em contrapartida, neste estudo, $75 \%$ dos participantes realizavam atividade física regular, o que pode ser um fator que influencia na correlação encontrada entre as variáveis em questão. Além disso, no presente estudo, utilizou-se um teste específico para a mensuração de força muscular dos extensores de joelho, enquanto no estudo de Atkins e Gardner ${ }^{2}$, realizou-se a mensuração da força desse mesmo grupo muscular por meio de um teste funcional, o que implica em um possível envolvimento de outras variáveis além da força muscular.

Nos resultados do presente estudo, não se observou correlação significativa entre o tempo gasto para a realização das cinco flexões plantares no teste de ponta de pé e a distância percorrida no TDBP. Gerdle et al..$^{22}$, avaliando a correlação entre força de flexores plantares na dinamometria isocinética e desempenho funcional no teste de esteira elétrica e no teste de caminhada livre, encontraram uma correlação de 0,61 e 0,89, respectivamente. Regensteiner et al. ${ }^{12}$, avaliando a força de gastrocnêmio com dinamômetro isocinético e desempenho, mensurado por teste em esteira elétrica, encontraram uma correlação entre essas variáveis de 0,41 . A fraca correlação encontrada por Regeinsteiner et al. ${ }^{12}$, quando comparada com a alta correlação observada no estudo de Gerdle et al. ${ }^{22}$, poderia ser explicada pelo fato de aqueles terem avaliado apenas uma porção da musculatura de tríceps sural, enquanto estes avaliaram o grupo muscular em questão em sua totalidade, sugerindo que pode existir influência da metodologia utilizada nos testes na força de associação das variáveis. No presente estudo, avaliou-se a força muscular de flexores plantares com teste de ponta de pé, teste que pode englobar outros fatores além da força da musculatura envolvida, influenciando, talvez, na inexistência da correlação. Além disso, como a maior parte dos participantes do presente estudo finalizou o TDBP por dor ou fadiga devido à CI, e o tempo gasto para a realização de cinco flexões plantares talvez não tenha sido suficiente para levar o indivíduo a tais sintomas, o teste de ponta do pé, como proposto, pode não ser o mais adequado para gerar correlação entre força e funcionalidade em pacientes com DAOP.

O presente estudo tem como limitações o tamanho da amostra e o percentual desigual de homens e mulheres avaliados. É preciso ter cautela na generalização dos resultados, considerando-se que o desempenho em testes de força muscular pode ser diferente entre homens e mulheres, e a relação entre produção de força e capacidade funcional pode ser distinta entre os sexos.

\section{Conclusão}

De acordo com os resultados obtidos no estudo, conclui-se que o desempenho funcional em um grupo de pacientes com DAOP, em sua maioria do sexo masculino e fisicamente ativos, foi fortemente correlacionado com a força muscular de extensores de joelho encontrada no teste de $1 \mathrm{RM}$, mas não com o desempenho observado pelo teste de ponta do pé com número de flexões plantares préestabelecido. Futuros estudos são necessários para avaliar se a força muscular de flexores plantares, mensurada de forma específica e isolada, correlaciona-se com função em pacientes com DAOP. 


\section{Referências}

1. McGuigan MR, Bronks R, Newton RU, et al. Muscle fiber characteristics in patients with peripheral arterial disease. Med Sci Sports Exerc. 2001;33:2016-21.

2. Atkins LM, Gardner AW. The relationship between lower extremity functional strength and severity of peripheral arterial disease. Angiology. 2004;55:347-55.

3. Cimminiello C.PAD. Epidemiology in pathophysiology. Thrombosis Research. 2002;106:295-301.

4. Novo S. Classification, epidemiology, risk factors, and natural history of peripheral arterial disease. Diabetes, Obesity and Metabolism. 2002;4:S1-S6.

5. Santos Filho MAL, Rosoky RMA, Costa DFF, Ferreira CB, Wolosker $\mathrm{N}$, Puech-Leão P. Comparação entre o resultado do tratamento clínico de pacientes com claudicação intermitente por obstrução femoropoplítea bilateral versus obstrução aórtica. J Vasc Bras. 2005;4:137-42.

6. Faxon DP, Fuster V, Libby P, et al. Atherosclerotic Vascular Disease Conference: Writing Group III: pathophysiology. Circulation. 2004;109:2617-25.

7. Gardner AW, Montgomery PS, Killewich LA. Natural history of physical function in older men with intermittent claudication. J Vasc Surg. 2004;40:73-8.

8. McDermott MM, Greenland P, Liu K, et al. The ankle brachial index is associated with leg function and physical activity: the Walking and Leg Circulation Study. Ann Intern Med. 2002;136:873-83.

9. Green S. Haemodynamic limitations and exercise performance in peripheral arterial disease. Clinical Physiology in Functional Imaging. 2002;22:81-91.

10. Scott-Okafor HR, Silver KKC, Parker J, Almy-Albert T, Gardner AW. Lower extremity strength deficits in peripheral arterial occlusive disease patients with intermittent claudication. Angiology. 2001;52:7-14

11. McDermott MM, Mehta S, Liu K, et al. Leg symptoms, the ankle brachial index, and walking ability in patients with peripheral arterial disease. J Gen Intern Med. 1999;14:173-81.

12. Regensteiner JG, Wolfel EE, Brass EP, et al. Chronic changes in skeletal muscle histology and function in peripheral arterial disease. Circulation. 1993;87:413-421.

13. McDermott MM, Guralnik JM, Albay M, Bandinelli S, Miniati, B, Ferrucci, L. Impairments of muscles and nerves associated with peripheral arterial disease and their relationship with lower extremity functioning: the InCHIANTI Study. J Am Geriatr Soc. 2004;52:405-10.
14. McDermott MM, Criqui MH, Greenland Pet, et al. Leg strength in peripheral arterial disease: Associations with disease severity and lower-extremity performance. I Vasc Surg. 2004; 39:523-30

15. Ritti-Dias RM, Wolosker N, de Moraes Forjaz CL, et al. Strength training increases walking tolerance in intermittent claudication patients: randomized trial. J Vasc Surg. 2010; 51:89-95.

16. McDermott MM, Ades P, Guralnik JM, et al. Treadmill exercise and resistance training in patients with peripheral arterial disease with and without intermittent claudication: a randomized controlled trial. JAMA. 2009;301:165-74.

17. Hiatt WR, Wolfel EE, Meier RH, Regensteiner JG. Superiority of treadmill walking exercise versus strength training for patients with peripheral arterial disease. Implications for the mechanism of the training response. Circulation. 1994; 90:1866-74.

18. Cunha-Filho IT, Pereira DAG, Carvalho AMB, Campedeli L, Sorares $M$, Freitas JS. Confiabilidade de testes de caminhada em pacientes claudicantes: estudo piloto. J Vasc Bras. 2008;7:106-111.

19. Pereira DAG, Oliveira KL, Cruz JO, Souza CG, Cunha-Filho IT. Avaliação da reprodutibilidade de testes funcionais na Doença Arterial Periférica. Revista Fisioterapia e Pesquisa. 2008;15:228-234.

20. Brown $M$, Sinacore DR, Host $H H$. The relationship of strength to function in the older adult. J Gerontol A Biol Sci Med Sci. 1995; 50:55-9.

21. Mc Dermott MM, Liu K, Greenland P, et al. Functional decline in peripheral arterial disease. Associations with the ankle brachial index and leg symptoms. JAMA. 2004;292:453-461.

22. Gerdle B, Hedberg B, Ãngquist KA, Fulg-Meyer AR. Isokinetic strength and endurance in peripheral arterial insufficiency with intermittent claudication. Scand J Rehabil Med. 1986; 18: 9-15.

Correspondência Danielle Aparecida Gomes Pereira Departamento de Fisioterapia da Universidade Federal de Minas Gerais Avenida Antônio Carlos, 6.627 - Pampulha CEP 31270-090 - Belo Horizonte (MG), Brasil E-mail: d.fisio@ig.com.br

Contribuições dos autores Concepção e desenho do estudo: DAGP, ICF Análise e interpretação dos dados: DAGP, BMAF, RAMG, VBFC, KOP, PSS, ICF Coleta de dados: DAGP, BMAF, RAMG, VBFC, KOP, PSS Redação do artigo: DAGP, BMAF, RAMG, VBFC, TPN Revisão crítica do texto: TPN, ICF Aprovação final do artigo: DAGP Análise estatística: DAGP, ICF Responsabilidade geral pelo estudo: DAGP Informações sobre financiamento: N/A* 